\title{
Comportamiento animal y composición botánica de la dieta de ovinos apacentando estrella africana (Cynodon plectostachyus (L.) Pers.) en monocultivo y asociada con Leucaena leucocephala (Lam.) de Wit. y Guazuma ulmifolia (Lam.)
}

\author{
Animal behavior and botanical composition of the diet of the african ovine star (Cynodon \\ plectostachyus (L) Pers.) in monoculture and associated with Leucaena leucocephala (Lam.) \\ de Wit. and Guazuma ulmifolia (Lam.)
}

\author{
René Pinto-Ruiz1, Angélica Anahí Pérez-Díaz ${ }^{*}$, David Hernández-Sánchez³, Francisco Guevara-Hernandez, \\ Gerardo Uriel-Trujillo 4 y Pedro Cadena-Iñiguez ${ }^{5}$
}

\begin{abstract}
${ }^{1}$ Facultad de Ciencias Agronómicas, Universidad Autónoma de Chiapas (UNACH). Chiapas, México. ${ }^{2}$ Estudiante del programa de Maestría en Ciencias en Producción Agropecuaria Tropical, Universidad Autónoma de Chiapas (UNACH). Chiapas, México.

${ }^{3}$ Colegio de Posgraduados, Montecillo. México, México. ${ }^{4}$ Facultad de Medicina Veterinaria y Zootecnia, Universidad Autónoma de Chiapas, Universidad Autónoma de Chiapas (UNACH). Chiapas, México. ${ }^{5}$ Instituto Nacional de Investigaciones Forestales Agrícolas y Pecuarias. México, México. *Correo electrónico: anny_chapa_93@hotmail.com
\end{abstract}

\section{RESUMEN}

Se realizó el presente estudio para conocer el comportamiento en pastoreo y la composición botánica de la dieta seleccionada por ovinos apacentando una pradera de "estrella africana" (Cynodon plectostachyus) en monocultivo y el mismo pasto asociado con Leucaena leucocephala y Guazuma ulmifolia, en dos épocas del año (a). Las variables obtenidas en un periodo de 12 horas (h) fueron: los tiempos de pastoreo, ramoneo, rumia y descanso bajo sol o bajo la sombra de los árboles, asimismo se obtuvo en un espacio de $24 \mathrm{~h}$, la frecuencia de orina y consumo de agua. Por otro lado, se midió la composición botánica de la dieta seleccionada por cada animal y la temperatura rectal de los ovinos apacentados en ambos tipos de praderas durante la época de sacas y de lluvias. Los resultados indicaron que el tiempo de pastoreo de los ovinos no fue modificado por el tipo de pradera en ambas estaciones del a. El tiempo de rumia fue mayor y el tiempo de descanso fue menor en los ovinos apacentando en la pradera arbolada. Se encontró que la temperatura corporal tomada por vía rectal disminuyó 0,25 y $0,38^{\circ} \mathrm{C}$ en la época seca y de lluvia, respectivamente, en relación a los animales que apacentaron en praderas en monocultivo. Los ovinos comieron más follaje arbóreo y bebieron más agua durante la época seca, asociado con mayor actividad de ramoneo. En conclusión, el sistema silvopastoril modifica favorablemente las actividades diarias de los ovinos en pastoreo y brinda mayor confort y bienestar a los animales.

Palabras clave: Conducta animal; dieta seleccionada; sistemas silvopastoriles; ovinos

\begin{abstract}
The present study was carried out to know the grazing behavior and the botanical composition of the diet selected by sheep grazing a meadow of "African star" (Cynodon plectostachyus) in monoculture and the same grass associated with Leucaena leucocephala and Guazuma ulmifolia, in two seasons of the year (yr). The variables obtained in a period of 12 hours (h) were: the times of grazing, browsing, rumination and rest under the sun or under the shade of the trees, also the frequency of urine and consumption was obtained in a space of $24 \mathrm{~h}$ of water. On the other hand, the botanical composition of the diet selected by each animal and the rectal temperature of the sheep grazed in both types of meadows during the sack and rain season were measured. The results indicated that the grazing time of the sheep was not modified by the type of pasture in both seasons of the yr. The rumination time was longer and the rest time was shorter in the sheep grazing in the wooded meadow. It was found that the body temperature taken by straight route decreased 0.25 and $0.38{ }^{\circ} \mathrm{C}$ in the dry and rainy season, respectively, in relation to the animals that grazed in monoculture meadows. Sheep ate more tree foliage and drank more water during the dry season, associated with higher browsing activity. In conclusion, the silvopastoral system favorably modifies the daily activities of grazing sheep and provides greater comfort and well-being to the animals.
\end{abstract}

Key words: Animal behavior; selected diet; silvopastoril systems; sheep 


\section{INTRODUCCIÓN}

La ganadería en el trópico seco del sureste de México se desarrolla bajo modelos de pastoreo, donde el consumo de gramíneas constituye la base de la alimentación de los animales $[27,28]$. En el sector campesino, la ovinocultura es parte integral de la estrategia de subsistencia socioeconómica y juega un papel central en la capitalización de esas familias pobres [20]. Los hatos ovinos (Ovis aries) se alimentan principalmente de recursos forrajeros pues su explotación es extensiva y se distribuyen en zonas marginadas [12]. Estas condiciones hacen necesaria la búsqueda de alternativas para mejorar la productividad del rebaño; ello significa la búsqueda de fuentes de alimentación más eficientes, de bajos costos y sostenibles en el uso de los recursos forrajeros.

Actualmente, en la región tropical de México existe interés en el desarrollo de sistemas de alimentación basados en sistemas silvopastoriles, en los cuales, los árboles forrajeros juegan un papel importante. Sin embargo, el manejo adecuado de los sistemas silvopastoriles depende mucho de la forma en que éstos son utilizados por los animales. Bajo estas condiciones, la integración de árboles con pastos resulta importante, pero hace complejo el sistema de producción debido a las interacciones planta-animal, ya que la presencia de arbóreas en las áreas de pastoreo puede hacer variar el comportamiento animal en respuesta a la sombra que proporcionan los árboles, a su estructura de copa, y a la cantidad y calidad del forraje disponible [37] en comparación con el tradicional pastoreo de praderas en monocultivo. Pocos son los estudios realizados con ovinos bajo estas circunstancias, por lo que el objetivo de este estudio fue conocer el comportamiento animal y la composición botánica de la dieta seleccionada por ovinos en apacentamiento de una pradera en monocultivo en comparación con pasturas en callejones.

\section{MATERIALES Y MÉTODOS}

\section{Localización del área de estudio}

La evaluación se realizó en el rancho La Trinidad, propiedad de un productor cooperante dedicado a la producción y comercialización de ganado ovino de registro. El rancho se localizó en el municipio Villaflores, al oeste del estado de Chiapas, México. Situándose entre los $16^{\circ} 13^{\prime} 15^{\prime \prime}$ de LN, 93 $13^{\circ}$ '07" LO, con altitud promedio de 610 metros sobre el nivel del mar. El clima es cálido subhúmedo con lluvias en verano; temperatura media anual de $24,3^{\circ} \mathrm{C}$ y precipitación pluvial media anual de 1.209 milímetros $(\mathrm{mm})$, distribuidas en verano, principalmente [10].

\section{Características de las praderas evaluadas}

La pradera silvopastoril consistió en la asociación de pastos Cynodon plectostachyus e Hyparrhenia ruffa con Leucaena leucocephala y Guazuma ulmifolia, bajo la modalidad silvopastoril conocida como pastura en callejones. Los árboles se encuentran sembrados en hileras sencillas y alternadas, orientadas de oriente a poniente, distancia entre hileras de tres metros $(\mathrm{m})$ y entre árboles de un $\mathrm{m}$. La altura de poda de los árboles fue 90 centímetros $(\mathrm{cm})$, con la finalidad de mantener disponible el material forrajero para el ramoneo de los animales. La edad de la pradera es de tres años (a) en una superficie de tres hectáreas (ha). La pradera de solo gramíneas consistió en un potrero establecido con pasto
C. plectostachyus, con cinco a de establecida y una superficie de cuatro ha.

Tanto en el sistema silvopastoril como en el de solo pasto se colocaron bebederos graduados para medir consumo de agua por los animales. Ambas praderas se manejaron bajo condiciones de temporal y sin fertilización.

\section{Características de los animales y su manejo}

Se utilizaron veinte ovinos machos de raza Pelibuey, con edad promedio de ocho meses (mes) y peso vivo de 24,6 $\pm 2,3$ kilogramos $(\mathrm{kg})$. Al inicio del experimento, los animales fueron desparasitados con Doramectina $\left(0,5\right.$ mililitros $(\mathrm{mL}) \cdot$ animal $\left.^{-1}\right)$ y vitaminados con Vitadel $\left(1,0 \mathrm{~mL} \cdot\right.$ animal $\left.^{-1}\right)$. En cada tratamiento (tipo de pradera) se asignaron diez ovinos. Los animales se marcaron con un número en el dorso para identificarlos. Cada cordero constituyó la unidad experimental (UP).

\section{Épocas de evaluación}

La conducta de los ovinos y composición de la dieta se evaluaron durante la época seca en los mes de marzo y abril, y para la época de lluvia fue en los mes de agosto y septiembre, ambas épocas del 2019 .

\section{Variables evaluadas}

\section{Conducta animal diaria y diurna}

Para el caso de la conducta diaria de los animales, se contemplaron periodos de observación continua de los ovinos en pastoreo durante 24 horas (h) durante los días (d) de marzo-abril y agosto-septiembre. En lo que respecta a la evaluación de la conducta diurna, los ovinos fueron observados durante $12 \mathrm{~h} \cdot \mathrm{d}^{-1}(6: 00$ - 18:00 h) durante los $\mathrm{d}$ de marzo-abril y agosto-septiembre. En ambos casos, se observó la conducta de los animales a intervalos de 10 minutos (min) a lo largo de todo el período de evaluación, anotando la actividad que cada animal realizaba. Para no influir en la conducta natural de los ovinos en pastoreo, se observaron los animales a $10 \mathrm{~m}$ de distancia, aproximadamente.

Las actividades diarias observadas en los ovinos en pastoreo fueron: tiempo de pastoreo, tiempo de ramoneo, tiempo dedicado a la rumia y descanso, número de veces orinando, número de veces que bebieron agua, y consumo diario de agua.

Para el caso de las actividades diurnas, se evaluaron las mismas variables en condiciones de sombra y a pleno sol. Cabe señalar que los bebederos en ambos sistemas estuvieron bajo sombra.

Con la finalidad de conocer los efectos de la cobertura arbórea sobre el animal, se midió cada h la temperatura rectal (TR) de cada animal utilizando un termómetro de mercurio (Brannan $\AA$, modelo BRA44-820-0, Thermometers and Instrumentation, Inglaterra) colocado directamente en el recto del animal.

\section{Composición botánica de la dieta}

Para determinar la composición botánica de la dieta se utilizó la técnica de microhistología de heces. El muestreo de heces se realizó en cada animal y cada época del a. Las muestras de heces fueron tomadas directamente del recto del animal en dos momentos: al entrar y al salir el ovino de la pradera correspondiente. Se realizó el montaje de patrones y heces [19]. 
Una vez identificadas las especies forrajeras consumidas por los animales, se procesaron individualmente para realizar el montaje de laminillas de referencia (patrones de cada especie forrajera). Del mismo modo se montaron laminillas temporales de las especies forrajeras presentes en las muestras fecales. Observando al microscopio (Velab®, modelo Ve-B50, Texas, Estados Unidos de América) las laminillas de referencia y las temporales, se realizó la descripción histológica para identificar estructuras anatómicas de las especies vegetales colectadas, tales como los tricomas, los cuales fueron descritos en su forma y tamaño.

\section{Diseño experimental y análisis estadístico}

Las variables de conducta diaria y diurna, así como la TR fueron analizadas mediante el procedimiento GLM de SAS [30]. Para ello, se utilizó un diseño experimental completamente al azar con dos tratamientos (tipo de pradera) y diez repeticiones por tratamiento (animales). Las medias de tratamientos fueron comparadas mediante la prueba de Tukey $(P<0,05)$. El análisis de los datos se realizó para cada época del a por separado.

\section{RESULTADOS Y DISCUSIÓN}

En la TABLA I se presentan los resultados del análisis estadístico realizado a las actividades de conducta de los ovinos pastoreando las praderas con solo C. plectostachyus y C. plectostachyus asociado con L. leucocephala y G. ulmifolia. Estos resultados muestran que la actividad de pastoreo fue similar $(P<0,05)$ en ambos tipos de praderas para cada época del a. Se aprecian diferencias estadísticas $(P<0,05)$ en el tiempo dedicado a descansar, ya que los ovinos pastoreando $C$. plectostachyus en monocultivo estuvieron descansando por más tiempo que aquellos que pastorearon la pradera asociada del pasto con arbóreas forrajeras, tanto en la época de secas como de lluvias. Sin embargo, el tiempo dedicado a la rumia fue mayor $(P<0,05)$ en los ovinos pastoreando la pradera asociada en ambas épocas del a. Del mismo modo, el tiempo dedicado al ramoneo fue mayor $(P<0,05)$ en ovinos que pastorearon la pradera del sistema silvopastoril durante la época de secas comparado con el mismo tipo de pradera durante la época de lluvias.

Los datos indican que los animales dedicaron más tiempo al pastoreo en la época seca $(8,93 \mathrm{~h})$ que durante el periodo de
Iluvia (6,68 h), lo cual podría explicarse por la disminución en la disponibilidad de biomasa forrajera en la estación seca del año, producto de falta de humedad, edad del pasto y al incremento de la estacionalidad de la producción de la biomasa [36], lo cual lleva al animal a emplear más tiempo al pastoreo para satisfacer sus necesidades de materia seca (MS) [25], lo que coincide con Solfanelli [31], quién evaluó el consumo de bovinos (Bos taurus) en pastoreo durante un a y reportó diferencias de hasta 100 min dedicados al pastoreo entre las épocas de seca y lluvia, sustituyendo que la falta de forraje en la época seca obliga a los animales a dedicar más tiempo al pastoreo en el intento de cubrir los requerimientos de MS debido, principalmente, al menor tamaño del bocado. Por otro lado, los promedios del tiempo dedicado al pastoreo fluctuaron entre 6,62 a $9,08 \mathrm{~h}$, tiempos que se encuentran dentro del rango reportado para rumiantes [25, 37, 40].

El tiempo dedicado al pastoreo por los ovinos durante la época Iluviosa en ambos tipos de praderas $(6,68 \mathrm{~h})$ fueron similares a los reportados por Zuccari y col. [40] en ovinos $(6,20 \mathrm{~h})$ para dos regímenes de pastoreo durante el verano. Por otro lado, los valores promedio del tiempo dedicado al pastoreo durante la época seca en ambos tipos de praderas $(8,93 \mathrm{~h})$ fueron mayores, aunque similares, a los reportados en otros trabajos con bovinos $[9,25,26]$.

La similitud entre los tratamientos indica que el uso de arbóreas en la pradera no influyó sobre el tiempo dedicado al pastoreo por los ovinos. Sin embargo, algunos trabajos reportan mayor tiempo de pastoreo en praderas con arbóreas $[22,24]$ ya que, los animales modifican sus estrategias de pastoreo en respuesta a presencia de árboles, así como a cambios en la fenología y disponibilidad del pasto presente bajo dosel arbóreo. Esta conducta es de suma importancia ya que al no modificarse el tiempo de pastoreo en ambos tipos de pradera se logra maximizar el consumo de pasto por el animal [35]. Adicionalmente, se debe considerar el tiempo que los animales dedican al ramoneo en la pradera asociada, lo cual suma mayor tiempo dedicado al consumo de forraje por parte de los ovinos.

La no diferencia entre ambos tipos de praderas obtenidas en el presente trabajo para el tiempo dedicado al pastoreo por los ovinos dentro de cada época del año $(P>0,05)$ se debe a los cambios en la disponibilidad del pasto basal ocasionado por la

TABLA I

Conducta de los ovinos pastoreando estrella africana (Cynodon plestotachyus) en monocultivo y asociada con Leucaena leucocephala y Guazuma ulmifolia durante dos épocas del año

\begin{tabular}{|c|c|c|c|c|c|c|}
\hline \multirow[b]{2}{*}{ Variables } & \multicolumn{3}{|c|}{ Época seca } & \multicolumn{3}{|c|}{ Época de Iluvias } \\
\hline & $\begin{array}{l}\text { Pradera asociada } \\
\text { horas·día-1/animal }^{-1}\end{array}$ & $\begin{array}{l}\text { Pradera solo } \\
\text { pasto }\end{array}$ & EEM & $\begin{array}{l}\text { Pradera Asociada } \\
\text { horas-día }^{-1} \text { animal }^{-1}\end{array}$ & $\begin{array}{c}\text { Pradera solo } \\
\text { pasto }\end{array}$ & EEM \\
\hline Pastoreo & $8,78^{a}$ & $9,08^{a}$ & 0,10 & $6,62^{a}$ & $6,75^{a}$ & 0,14 \\
\hline Descanso & $7,28^{b}$ & $9,55^{a}$ & 0,44 & $7,55^{b}$ & $11,63^{a}$ & 0,69 \\
\hline Rumia & $6,08^{a}$ & $5,37^{b}$ & 0,14 & $7,28^{a}$ & $5,57^{b}$ & 0,33 \\
\hline Ramoneo & $2,40^{a}$ & -- & & $1,85^{b}$ & -- & 0,13 \\
\hline
\end{tabular}

Letras distintas en la misma hilera dentro de cada época indican diferencias estadísticas $(\mathrm{P}<0,05)$; EEM = Error estándar de la media. 
poda en los árboles, existen otras variables que puedan afectar el tiempo dedicado al pastoreo, las cuales son el microclima generado por las copas de los árboles [2].

Para los tiempos de descanso y rumia, los animales de ambos tratamientos dedicaron mayor tiempo a ambas actividades durante las lluvias $(9,59$ y $6,42 \mathrm{~h}$, respectivamente) que durante la época seca ( 8,54 y $5,72 \mathrm{~h}$, respectivamente), debido a que dedicaron más tiempo al pastoreo $(6,68$ y 8,3 h para le época de lluvia y seca, respectivamente).

El tiempo en que los animales no están comiendo, rumiando o ingiriendo agua, es definido como ocio o descanso. La tendencia a mayor tiempo dedicado al descanso $(P<0,05)$ fue para la pradera en monocultivo, lo cual coincide con lo reportado por Espinoza y col. [9] en bovinos pastoreando praderas en monocultivo y asociadas en sistemas silvopastoriles. Sin embargo, el tiempo promedio de descanso encontrado en ambos tipos de praderas es mayor que el promedio reportado en trabajos anteriores, previamente citados.

El tiempo de pastoreo que dedicaron los ovinos $(P<0,05)$ en la pradera con monocultivo presentaron diferencias significativas en con comparación con los animales asignados a la pradera silvopastoril, compensado con mayor tiempo dedicado a la rumia $(P<0,05)$. Al respecto, Arnold y Dudzinsky [4] mencionan que después del pastoreo, la actividad que mayor tiempo dedican los rumiantes es a la rumia (5 a $9 \mathrm{~h}$ ).

El tiempo que los ovinos dedicaron a la rumia mostró la misma tendencia que otras investigaciones han reportado Espinoza y col. [9] con mayores tiempos de rumia en los animales que pastorean praderas silvopastoriles en comparación con animales pastando praderas en monocultivo.

Al considerar la importancia de las diferentes actividades realizadas por los ovinos pastoreando en monocultivo de gramíneas y en sistemas silvopastoriles, los animales dedican el 60 y $55 \%$ del tiempo al pastoreo y a la rumia, respectivamente, lo cual coincide con lo reportado por Sotckdale y King [34] y Hodgson [15].

La actividad de ramoneo consiste en la remoción de hojas y tallos tiernos ricos en proteína directamente de la planta [6]. En este sentido, el tiempo dedicado al ramoneo fue mayor en la época seca (TABLA I), este comportamiento pudo deberse a que en esta época existió menor disponibilidad y baja calidad del pasto, lo que motivó a los animales a aumentar el tiempo del ramoneo para satisfacer sus necesidades alimenticias. Este comportamiento también se ha reportado con bovinos [26]. En este trabajo, el tiempo dedicado al ramoneo fue menor $(1,48$ vs $0,38 \mathrm{~h}$, para la época seca y lluviosa, respectivamente) a las observadas en el presente estudio $(2,40$ vs $1,85 \mathrm{~h}$, para las épocas de secas y lluviosas, respectivamente). Lo anterior explica la naturaleza de los pequeños rumiantes de ramonear más que los bovinos [18]. Respuestas similares en vaquillas fueron reportaras en los trabajos de Espinoza y col. [9].

El tiempo dedicado al ramoneo representó el 10 y $7,7 \%$ del total de las actividades diarias de los ovino en pastoreo para las épocas seca y lluviosa, respectivamente. Analizando la misma variable, Betancourt y col. [6] reportaron que las vacas dedicaron 7,75 \% del tiempo total de pastoreo al ramoneo de especies arbóreas, lo cual fue similar al tiempo que dedicaron los ovinos al ramoneo en la presente investigación. Sin embargo, los valores promedios reportados por Espinoza y col. [9] fueron menores (2\%) para vaquillas pastoreando en bosque natural.

Cabe señalar que, aunque no existieron diferencias $(P>0.05)$ entre el tiempo de pastoreo en las praderas en monocultivo y asociadas, numéricamente fue mayor en animales que apacentaron en praderas asociadas, así mismo, estos animales distribuyeron el tiempo en realizar otras actividades como ramoneo (27\%). Este resultado fue similar al encontrado en bovinos en experimentos similares (25\%) [28].

El mayor tiempo dedicado por los ovinos al ramoneo de las arbóreas $(P<0,05)$ durante la época seca puede estar relacionado con el forraje seco del estrato herbáceo presente en la pradera [26], ya que los animales tienen preferencia por la biomasa verde durante la época seca [38]. En este sentido, los árboles en los potreros proporcionan forraje verde y de buena calidad en la época seca, cuando la biomasa disponible de los pastos es de baja calidad [14].

En lo que respecta a la conducta de las actividades diurna de los ovinos pastoreando ambos tipos de praderas por $12 \mathrm{~h}$ durante la época seca (TABLA II), reveló que el pastoreo como el ramoneo se realizaron indistintamente $(P<0,05)$ tanto en condiciones de sol como de sombra. Sin embargo, el tiempo dedicado por los ovinos a descanso y rumia fue mayor bajo la sombra que a pleno

TABLA II

Conducta diurna de los ovinos pastoreando por 12 horas estrella africana (Cynodon Plestostachyus) en monocultivo y asociada con Leucaena leucocephala y Guazuma ulmifolia, durante dos épocas del año

\begin{tabular}{ccccccc}
\hline \multirow{2}{*}{$\begin{array}{c}\text { Variables } \\
\text { (horas diurnas) }\end{array}$} & Sol & Sombra & EEM & Sol & Sombra & EEM \\
\cline { 2 - 7 } & $3,40^{\mathrm{a}}$ & $3,47^{\mathrm{a}}$ & 0,09 & $0,67^{\mathrm{b}}$ & $3,60^{\mathrm{a}}$ & 0,49 \\
Pastoreo & $0,13^{\mathrm{b}}$ & $1,88^{\mathrm{a}}$ & 0,31 & $0,30^{\mathrm{b}}$ & $2,40^{\mathrm{a}}$ & 0,35 \\
Descanso & $0,30^{\mathrm{b}}$ & $1,23^{\mathrm{a}}$ & 0,17 & $0,35^{\mathrm{b}}$ & $2,83^{\mathrm{a}}$ & 0,41 \\
Rumia & $0,65^{\mathrm{a}}$ & $0,93^{\mathrm{a}}$ & 0,10 & $1,52^{\mathrm{a}}$ & $0,48^{\mathrm{b}}$ & 0,17 \\
\hline
\end{tabular}

Letras distintas en la misma hilera dentro de cada época indican diferencias estadísticas $(P<0,05)$; EEM = Error estándar de la media. 
sol $(P<0,05)$. Por otro lado, en la época lluviosa, los animales dedicaron mayor tiempo $(P<0,05)$ a pastorear, descansar y rumiar bajo la sombra, mientras que el ramoneo fue realizado por más tiempo a pleno sol $(P<0,05)$.

La presencia de árboles en los potreros crea un microclima con efectos positivos en la conducta del animal, lo cual explica el mayor tiempo que los ovinos dedicaron al descanso y rumia bajo la sombra de la pradera con árboles en ambas épocas del año, lo cual puede explicarse debido a que los animales prefieren realizar las actividades de descanso y rumia en condiciones de tranquilidad y confort $[23,24]$.

El tiempo diurno dedicado al pastoreo presentó una inferencia de $28 \%$ bajo sol y $28.9 \%$ bajo sombra, mientras que el tiempo de descanso y rumia a pleno sol fue de 3,6 y $26,0 \%$ bajo sombra, similares a los reportados anteriormente para ovinos pastoreando $P$. saman y G. ulmifolia durante época seca [39]. Por otro lado, los animales que estuvieron mayor actividad bajo sombra que en las praderas sin presencia de cobertura vegetal $(7,51 \mathrm{~h}$ y $5,3 \mathrm{~h}$, respectivamente), lo cual confirma la importancia del dosel arbóreo como aportador de sombra para los animales [41].

La composición botánica de la dieta de los ovinos (TABLA III) fue más variada debido a la mayor diversidad de especies forrajeras en la pradera con árboles; sin embargo, la proporción de gramíneas en la dieta fue siempre más alta, tanto en la época de secas como de lluvias, en una relación de 81,77 vs $18,20 \%$, entre el forraje proveniente de las gramíneas y de las arbóreas, respectivamente. La presencia de forraje proveniente de leñosas en la dieta de los ovinos durante las dos épocas del año podría deberse a que el follaje de estas especies está disponible durante todo el año y es de alto valor nutricional [11, 40]. Dentro de las arbóreas, L. leucocephala fue la especie forrajera más ingerida por ovinos con 14.74 y $10.06 \%$ en la época seca y lluvia, respectivamente, en comparación con G. ulmifolia (5.36 y $6.25 \%$ en la época seca y lluvia, respectivamente).

Se ha documentado que incluir follaje arbóreo mejora la calidad nutricional de la dieta consumida por los animales en pastoreo, en esta investigación, el $20 \%$ de la dieta estuvo constituida por follaje de las arbóreas en la época seca y $16,25 \%$ en la época de lluvias. Estos resultados son similares a los reportados en otros trabajos con bovinos, en los cuales, el $16,1 \%$ de la dieta estaba compuesta por follaje arbóreo [7], 18,1 \% cuando los bovinos pastoreaban en acahuales [29] y $26,5 \%$ cuando toretes pastorearon en vegetación natural [13].

En lo que respecta al pastoreo en áreas de vegetación secundaria (acahuales), las arbustivas son parte importante de la composición de la dieta en la época de menor precipitación, y se estima que más del $60 \%$ de la materia seca consumida por los bovinos proviene de arbustivas que se establecen en los acahuales [13, 32]. Esta respuesta puede estar asociada a que las arbóreas, a diferencia de las gramíneas, ofrecen material verde durante la época seca que es consumido por los animales [14]. Asimismo, se ha determinado un incremento en el consumo de follaje de los arbustos a medida que el pasto comienza a madurar [7].

La selección entre las gramíneas y las arbóreas por los ovinos puede asociarse con la preferencia o palatabilidad del recurso forrajero o con la abundancia de las especies forrajeras en la pradera, así como con la carga animal y el tiempo de pastoreo empleados. Con relación a la gramínea C. plestostachyus, los resultados indican la presencia de Hyparrhenia ruffa, lo que indica la selectividad de los animales cuando tienen la posibilidad de seleccionar el forraje ofrecido.

Aunque la técnica microhistológica es útil para determinar las diferentes especies vegetales que componen la dieta, la información proporcionada mediante esta técnica no es suficiente para estimar el consumo de las diferentes plantas forrajeras presentes en una pradera. Los resultados resaltan la importancia de promover mayor diversidad vegetal en las áreas de pastoreo. Esto permitirá a los animales variar su dieta y mejorar la calidad de la misma resultando en un mejor comportamiento animal.

El análisis estadístico de la influencia del tipo de pradera en la temperatura rectal de los ovinos durante la época seca (TABLA IV) indica que no hubo diferencias entre los ovinos asignados a la pradera en monocultivo y aquellos que pastorearon la pradera con estrella africana asociada con arbóreas, mientras que, durante la época de lluvias, la temperatura rectal fue mayor $(P<0,05)$ en los ovinos pastoreando la pradera sin árboles.

El rango de temperatura rectal de los ovinos en pastoreo $(38,41$ a $\left.39,61^{\circ} \mathrm{C}\right)$ estuvo dentro de los límites fisiológicos adecuados $(37,8$ a $40,0^{\circ} \mathrm{C}$ ) reportados por Arias y col. [3] aunque el promedio de TR de los ovinos pastoreando la pradera en monocultivo durante la época lluviosa superó ligeramente este rango. La TR promedio de los ovinos fue de $38,5^{\circ} \mathrm{C}$ para ambos tipos de praderas durante

TABLA III

Composición botánica (\%) de la dieta seleccionada por ovinos pastoreando estrella africana (Cynodon plestostachyus) en monocultivo y asociada con Leucaena leucocephala y Guazuma ulmifolia, durante dos épocas del año

\begin{tabular}{cccccc}
\hline \multirow{2}{*}{ Nombre Común } & Nombre Científico & \multicolumn{2}{c}{ Época seca } & \multicolumn{2}{c}{ Época de Iluvias } \\
\cline { 3 - 6 } & $\begin{array}{c}\text { Pradera } \\
\text { asociada }\end{array}$ & $\begin{array}{c}\text { Pradera } \\
\text { solo pasto }\end{array}$ & $\begin{array}{c}\text { Pradera } \\
\text { asociada }\end{array}$ & $\begin{array}{c}\text { Pradera } \\
\text { solo pasto }\end{array}$ \\
\hline Estrella Africana & Cynodon plestostachyus & 76,04 & 100 & 73,06 & 93,24 \\
Jaragua & Hyparrhenia ruffa & 3,84 & 10,60 & 6,75 \\
Leucaena & Leucaena leucocephala & 14,74 & 10,06 & 6,25 \\
\hline
\end{tabular}


TABLA IV

Temperatura rectal (TR) de ovinos pastoreando estrella africana (Cynodon plestostachyus) en monocultivo y asociada con Leucaena leucocephala y Guazuma ulmifolia durante dos épocas del año

\begin{tabular}{ccccccc}
\hline \multirow{2}{*}{ Variables } & \multicolumn{3}{c}{ Época seca } & \multicolumn{3}{c}{ Época de lluvias } \\
\cline { 2 - 7 } & $\begin{array}{c}\text { Pradera } \\
\text { Asociada }\end{array}$ & $\begin{array}{c}\text { Pradera } \\
\text { solo pasto }\end{array}$ & EEM & $\begin{array}{c}\text { Pradera } \\
\text { Asociada }\end{array}$ & $\begin{array}{c}\text { Pradera } \\
\text { solo pasto }\end{array}$ & EEM \\
\hline TR & $38,41^{\mathrm{a}}$ & $38,66^{\mathrm{a}}$ & 0,10 & $39,23^{\mathrm{b}}$ & $39,61^{\mathrm{b}}$ & 0,08 \\
\hline
\end{tabular}

Letras distintas en la misma hilera dentro de época indican diferencias estadísticamente significativas $(P<0,05)$; TR $=$ Temperatura rectal en grados Celsius; EEM = Error estándar de la media

la época de seca, siendo ligeramente inferior a la TR promedio reportada por Alzina y col. [1] en bovinos pastoreando en la zona oriente de Yucatán durante la misma época del año (TR de $39,4^{\circ} \mathrm{C}$ ), pero fue similar a la reportada por Betancourt y col. [6] en vacas pastoreando potreros con baja cobertura arbórea durante la época de sequía (TR de $38,4^{\circ} \mathrm{C}$ ).

En este trabajo no se encontraron diferencias $(P>0,05)$ para la TR de los ovinos que pastorearon ambos tipos de praderas durante la época seca. Estos resultados contrastan con los obtenidos por Betancourt y col. [6] quienes reportaron diferencias significativas en la TR a favor de las vacas que pastorearon la cobertura arbórea. Por otro lado, la TR de los ovinos que pastorearon bajo sombra de los árboles fue mayor $(P<0,05)$ durante la época lluviosa, lo cual pudo estar asociado a mayor humedad ambiental $(79,95$ vs $63,53 \%$ para las épocas de lluvias y secas, respectivamente). En este sentido, $70 \%$ de humedad ambiental es considerada aceptable para los ovinos, siempre y cuando la temperatura ambiental esté dentro de la zona termoneutral $\left(10\right.$ a $27^{\circ} \mathrm{C}$ [17]. Se sabe que la relación entre la temperatura y humedad ambiental son importantes en al balance calórico del animal [21].

En este trabajo, la temperatura ambiental en las praderas fue: $26,36^{\circ} \mathrm{C}$ en la época lluviosa y $26,00^{\circ} \mathrm{C}$ en la época seca. Durante la época de lluvias, la TR fue menor $(P<0,05)$ fue menor en los ovinos pastoreando la pradera asociada $\left(39,23{ }^{\circ} \mathrm{C}\right)$, comparado con aquellos animales que pastorearon la pradera de solo pasto $\left(39,61^{\circ} \mathrm{C}\right)$. Estas diferencias pueden asociarse con la influencia de la cobertura arbórea que pudo disminuir la TR en los animales creando un ambiente favorable para los ovinos [5].
Lo anterior es importante ya que en condiciones de estrés calórico el consumo de pasto disminuye [33] lo cual es más acentuado al disminuir del tiempo dedicado al pastoreo como sucedió en este trabajo. Sin embargo, la TR de los ovinos pastoreando en el sistema silvopastoril mostró tendencia a ser menor en ambas épocas del año comparado con la TR de los ovinos pastoreando la pradera en monocultivo. Esto puede explicarse por al hecho de que la presencia de árboles en la pradera tiene alto potencial para disminuir el estrés calórico en los animales, Mahecha y col. [16] y Souza y col. [33] aseguran que los árboles, a través de la sombra, contrarrestan la intensidad de los factores climáticos, pues interfieren parcialmente el paso de la radiación solar hacia la superficie corporal del animal, disminuyendo el estrés térmico y creando condiciones de confort.

En cuanto a la influencia del tipo de pradera sobre las conductas de los ovinos asociadas al clima, los resultados revelan promedios mayores $(P<0,05)$ en la frecuencia de evacuación de orina $(F E O)$, frecuencia en beber agua (FBA) y consumo diario de agua (CTA) en los ovinos pastoreando la pradera en monocultivo durante la época seca (TABLA V). Asimismo, hubo diferencias significativas $(P<0,05)$ en FBA en los ovinos pastoreando la pradera en monocultivo durante la época de lluvias. De este modo, los ovinos orinan más veces, acceden a la fuente de agua y consumen más agua durante la temporada de secas que durante la estación lluviosa (32,15 vs 29,53 veces; 7,40 vs 2,57 veces y 5,12 vs 0,56 litros (L), respectivamente). Arnold y Dudzinsky [4] indican que, además de los factores ambientales, el tipo de forraje y la distribución de la fuente de agua influyen en la frecuencia con que los animales toman agua. Sin embargo, los resultados del presente estudio se asocian

TABLA V

Actividades diarias de ovinos pastoreando estrella africana (Cynodon Plestostachyus) en monocultivo y asociada con Leucaena leucocephala y Guazuma Ulmifolia, en dos ëpocas a lo largo del año

\begin{tabular}{ccccccc}
\hline & \multicolumn{3}{c}{ Seca } & & \multicolumn{2}{c}{ Lluvia } \\
\cline { 2 - 6 } Variables & $\begin{array}{c}\text { Pradera } \\
\text { Asociada }\end{array}$ & $\begin{array}{c}\text { Pradera } \\
\text { solo pasto }\end{array}$ & EEM & $\begin{array}{c}\text { Pradera } \\
\text { Asociada }\end{array}$ & $\begin{array}{c}\text { Pradera } \\
\text { solo pasto }\end{array}$ & EEM \\
\hline FDO & $30,50^{\mathrm{b}}$ & $33,80^{\mathrm{a}}$ & 0,40 & $28,71^{\mathrm{a}}$ & $30,36^{\mathrm{a}}$ & 0,10 \\
FBA & $6,10^{\mathrm{b}}$ & $8,69^{\mathrm{a}}$ & 0,44 & $1,14^{\mathrm{b}}$ & $4,07^{\mathrm{a}}$ & 0,14 \\
$\begin{array}{c}\text { CTA, } \\
\text { litros.animal-1.dia-1 }\end{array}$ & $4,37^{\mathrm{b}}$ & $5,87^{\mathrm{a}}$ & 0,14 & $0,28^{\mathrm{a}}$ & $0,99^{\mathrm{a}}$ & 0,14 \\
\hline
\end{tabular}

Letras distintas en la misma hilera dentro de cada época indican diferencias estadísticamente significativas $(P<0,05)$; FDO = Frecuencia de orinar; FBA = Frecuencia de beber agua; $C T A=$ Consumo total de agua; EEM = Error estándar de la media. 
más a la condición del forraje, ya que la temperatura ambiente durante el estudio fue muy similar en ambas épocas del a (26,36 y $26,00^{\circ} \mathrm{C}$ para la época seca y lluviosa, respectivamente).

Lo ocurrido en la conducta diaria de los ovinos en la época seca está asociada a los beneficios de confort que los árboles de la pradera asociada ofrecen al animal y no a la temperatura ambiente o a la calidad del forraje, pues ésta fue similar en ambos tipos de praderas. De este modo, la presencia de árboles de sombra puede reducir la pérdida de líquidos corporales que provoca la radiación solar en los ovinos, contribuyendo a disminuir los requerimientos de agua de los animales. Estudios previos han reportado mayores consumos de agua durante los periodos calurosos por mayores pérdidas de líquidos corporales comparado con menor consumo de agua durante los periodos fríos y húmedos [8].

Durante la época de lluvias los ovinos pastoreando la pradera en monocultivo fueron más veces a tomar agua $(P<0,05)$, sin embargo, la cantidad de agua ingerida en cada toma fue moderada ya que el consumo total de agua diariamente por los ovinos fue similar entre ambos tratamientos $(P<0,05)$. Por tanto, la presencia de árboles en los potreros minimiza el consumo de agua y maximiza las actividades diarias de los ovinos.

\section{CONCLUSIONES}

Los resultados indican que el tiempo que los ovinos dedican al pastoreo no es una actividad animal que sea modificada por la cobertura arbórea presente en el sistema silvopastoril conformado por Cynodon plectostachyus en asociación con Leucaena leucocephala y Guazuma ulmifolia, aun cuando el tiempo de descanso y de rumia sean menores en las áreas de pastoreo arboladas, comparado con el pastoreo de praderas en monocultivo a pleno sol. Esta conducta de los ovinos puede asociarse al confort proporcionado por la sombra de los árboles, sobre todo en la época del año de mayor temperatura y humedad ambiental (estación lluviosa), en la cual la temperatura rectal es menor en los ovinos que pastorean en praderas asociadas.

El estudio de la composición de la dieta muestra que los ovinos comieron más follaje arbóreo durante la época seca en comparación con el consumo de arbóreas durante la época de lluvias, lo cual pudo estar asociado a mayor tiempo dedicado al ramoneo en esta época del año.

Finalmente, la mayor frecuencia en orinar durante la época seca debe relacionarse con mayor consumo de agua por pérdidas de agua corporal durante la época seca en los ovinos pastoreando la pradera en monocultivo, mientras que la menor frecuencia de la actividad de orinar en los ovinos que pastorearon la pradera asociada puede deberse a condiciones de confort ambiental proporcionado por la sombra de los árboles en el sistema silvopastoril.

\section{REFERENCIAS BIBLIOGRÁFICAS}

[1] ALZINA-LÓPEZ, A.; FARFÁN-ESCALANTE, J. C.; VALENCIAHEREDIA, E.; YOKOYAMA-KANO, J. Condición ambiental y su efecto en la temperatura rectal y frecuencia respiratoria en bovinos cruzados (Bos taurus $x$ Bos indicus) del estado de Yucatán, México. Rev. Biomed. 12 (2):112-121. 2001.
[2] AMAYA, G.Y.; CABALLERO, C.P.; LUNA, R. E.; SÁNCHEZ, M. F.; MANZANILLA, Q. G.; VILLALÓN, M.H. Evaluación del microclima en un sistema silvopastoril en Montemorelos, Nuevo León, México. Recursos Genéticos Forestales. Forest. Verac. 21 (1):19-22. 2019.

[3] ARIAS, R.A.; MADER, T. L.; ESCOBAR, P. C. Factores climáticos que afectan el desempeño productivo del ganado bovino de carne y leche. Temuco, Chile. Arch. Med. Vet. 40 (1): 7-22. 2008.

[4] ARNOLD, G. W.; DUDZINSKI, M. L. Ethology of free-ranging domestic animals. In: Arnold, G.W.; Dudzinski, M.L. (Eds). Developments in animal and veterinary sciences. Elsevier/ North-Holland Amsterdam. New York. Pp 179-192. 1978

[5] BARRAGÁN, H. W.; MAHECHA, L. L.; CAJAS, G. Y. Variables Fisiológicas-Metabólicas de Estrés Calórico en Vacas Bajo Silvopastoreo y Pradera sin Árboles. Rev. Agron. Meso. Amer. 26 (2):211-223. 2015.

[6] BETANCOURT, K.; IBRAHIM, M.; VILLANUEVA, C.; VARGAS, B. Efecto de la cobertura arbórea sobre el comportamiento animal en fincas ganaderas de doble propósito en Matiguás, Matagalpa, Nicaragua. Livest. Res. Rural. Develop. 17 (7): 1-18. 2005.

[7] CHÁVEZ, A.; IERRO, G.; PEÑA, H.R.; SÁNCHEZ, E.; ORTIZ, V. Composición botánica y valor nutricional de la dieta de bovinos en un pastizal mediano abierto en la región central de Chihuahua. Tec. Pec. Mex. 50: 9-105. 1986.

[8] ECHAVARRÍA, A.; MIAZZO, R. El ambiente en la producción animal. 2002. Adaptación, Aclimatación, Estrés, y Clima. Sitio Argentino de producción animal. Facultad de Agronomía y Veterinaria, Universidad Nacional de Río Cuarto. Córdoba, Argentina. En línea: https://bit.ly/3wH03oN.12-05-2021.

[9] ESPINOZA, F.; HERNANDEZ, R. A.; FOLACHE, L. Etología de vaquillas doble propósito en un sistema silvopastoril durante el periodo seco en una Sabana tropical. Instituto Nacional de Investigaciones Agrícolas. Centro Nacional de Investigaciones Agropecuarias. Venezuela. Zoot. Trop. 26 (4):429-437. 2008.

[10] GARCÍA, E. Distribución de los grupos climáticos de Köppen en México. Modificaciones al sistema de clasificación climática de Köppen, 3th. Ed. Universidad Nacional Autónoma de México. Pp 252. 1989.

[11] GENIN, D.; CROCHOT, C.; MSOU, S.; ARABA, A.; ALIFRIQUI, M. Meadow up a tree: Feeding flocks with a native ash tree in the Moroccan mountains. Pastoralism: Res. Pol. Pract. 6 (11): 1-12. 2016.

[12] GÓNGORA, P. R.; GÓNGORA, G. S.; MAGAÑA, M. M.; LARA. L. P. Caracterización técnica y socioeconómica de la producción ovina en el estado de Yucatán, México. Agron. MesoAmer. 21 (1): 131-144. 2010.

[13] HERNÁNDEZ, L.A. Conducta Ingestiva y Comportamiento Animal en Vegetación Multiestrata y Praderas de Gramíneas. Villaflores, Chiapas; Universidad Autónoma de Chiapas. Tesis de Grado. Pp 150. 2003. 
[14] HERNÁNDEZ, H. M.; LÓPEZ, O. S.; JARILLO, R. J.; ORTEGA, J. E.; PÉREZ, E. S.; DÍAZ, R. P.; CROSBY, G. M. Rendimiento y calidad nutritiva del forraje en un sistema silvopastoril intensivo con Leucaena leucocephala y Megathyrsus maximus Cv. Tanzania. Rev. Mex. Cien. Pec. 11 (1):53-69. 2020.

[15] HODGSON, J. Longman Handbooks in Agriculture, Essex. Grazing management: science into practice. Pp. 203. 1990.

[16] JOHNSON, H.D. Bioclimatic effects on grout reproduction and milk production of livestock. In: Johnson, H. D. (Ed). Bioclimatology and adaptation of livestock. Amsterdam: Elsevier. Pp. 1-31. 1987.

[17] MAHECHA, L.; DURÁN, C.V.; ROSALES, M.; MOLINA, C. H. Grazing, browsing time and milk production of lucerna cows in a silvopastoral system in different seasons of the year. In: International Symposium on silvopastoral systems 2. Congress on Agroforestry and Livestock Production in Latin America. San José, April 2-9, Costa Rica, Pp 11-18. 2001.

[18] LEMA, M.; FELIX, A.; DORSEY, J. Lamb production and forage quality under a forage system consisting of AU Triumph tall fescue and Tifton 44 Bermuda grass. Small. Rum. Res. 37 (3):249-253. 2000.

[19] LÓPEZ, A.; PINTO, R.; GUEVARA, F.; MEDINA, F.; HERNÁNDEZ, D.; ORTEGA, L. Descripción de la técnica. La microhistología y su aplicación en la agroforestería pecuaria. Editorial Fontamara. $1^{\text {st }}$.Ed. México. 127 pp. 2017.

[20] MARTÍNEZ, G. E.; MUÑOZ, R. M.; GARCÍA, M. J.; SANTOYO, C. V.; ALTAMIRANO, C. J.; ROMERO, M. C. El fomento de la ovinocultura familiar en México mediante subsidios en activos: lecciones aprendidas. Agron. MesoAmer. 22 (2):367-377. 2011.

[21] MOUNT, L.E. Adaptation to thermal environment. Man, and his productive animals. Edward Arnold (Publishers). London. Pp. 13-333. 1979.

[22] NARANJO, J.F.; RIVERA, J.E.; CUARTAS, C.A. Efecto de la oferta y el consumo de Tithonia diversifolia en un sistema silvopastoril intensivo (SSPI), en la calidad y productividad de leche bovina en el piedemonte Amazónico colombiano. Colombia. Livest. Res. Rural. Develop. 27 (10): 1-13. 2015.
[23] ORJUELA-CHAVES, J. A.; CUBILLOS-RODRIGUEZ, J. M.; ORRES-SILVA, O. Evaluación del comportamiento de bovinos de doble propósito bajo diferentes niveles de cobertura arbórea en departamento del Caquetá - Colombia. Universidad de la Amazonia, Florencia - Caquetá. Rev. Fac. Cien. Agrop FAGROPEC.7 (1): 60-64. 2015.

[24] PARRA, H. J.; ESTRADA, C.; GLORIA, E.; PARRA, F. D.; MONTEALEGRE, V. N. Efecto de diferentes sistemas enriquecidos sobre el comportamiento de bovinos doble propósito en producción lechera en la amazonia colombiana. Rev. Electrón. Vet. REDVET. 18 (12): 1-22. 2017.

[25] PATIÑO, P. R.; GONZÁLEZ, M. K.; PORRAS, S. F.; SALAZAR R, L.; VILLALBA, S. C.; BETANCOURT, J. G. Comportamiento ingestivo diurno y desempeño de novillos en pastoreo pertenecientes a tres grupos genéticos durante dos épocas climáticas. Livest. Res. Rural Develop. 20 (36): 15-23. 2008.

[26] PINTO, R. R.; ORTEGA, R. L.; GÓMEZ, C. H.; GUEVARA, H.F.; HERNÁNDEZ, S. D. Comportamiento animal y características de la dieta de bovinos pastoreando estrella africana sola y asociada con árboles. Rev. Mex. Cien. Pec. 5 (3): 365-374. 2014.

[27] QUERO, C. A.; ENRÍQUEZ, Q. J.; MIRANDA, J. L. Evaluación de especies forrajeras en América Tropical, avances o status quo. Caracas. Venezuela. Intercien. 32 (8): 566-571. 2007.

[28] QUERO, C. A.; MIRANDA, J.; VILLANUEVA, A. J. Recursos genéticos de gramíneas para el pastoreo extensivo. Condición actual y urgencia de su conservación ante el cambio climático. Avances en Investigación Agropecuaria - AIA. Rev. Invest. Difus. Cien. Agrop. 21 (3): 63-85. 2017.

[29] SANSORES, L. I. Composición botánica y valor nutricional de la dieta de bovinos en un área de vegetación secundaria en Quintana Roo. Inst. Nac. Invest. Fores. Agrí. Pec. México. Pp 105 - 117. 1999.

[30] STATISTICAL ANALYSIS SYSTEM INSTITUTE (SAS). SAS User Versión 4.0 .1991.

[31] SOLFANELLI, P. Consumo de bovinos en pastoreo. Rev. Soc. Rural de Jesús María, Córdoba. 114: 22-33. 2002. 
[32] SOSA, R.E.; PÉREZ, R. D.; ORTEGA, R. L.; ZAPATA, G. Evaluación del potencial forrajero de árboles y arbustos tropicales para la alimentación de ovinos. Tec. Pec. Mex. 42 (2):129-144. 2004.

[33] SOUZA, D. E.; ABREU, M.; IBRAHIM, M.; HARVEY, C.; JIMÉNEZ, F. Caracterización del componente arbóreo en los sistemas ganaderos de La Fortuna de San Carlos, Costa Rica. Agrofor. Amer. 7 (26): 53-56. 2000.

[34] STOCKDALE, C.; KING, K. Effect of stocking rate on the grazing behaviour and faecal output of lactating dairy cows. Grassland and Forage Sci. 38: 215-218. 1983.

[35] TARAZONA, A.M.; CEBALLOS, M.C.; NARANJO, J.F.; CUARTAS, C.A. Factores que afectan el comportamiento de consumo y selectividad de forrajes en rumiantes. Rev. Colomb. Cien. Pec. 25 (3): 473-487. 2012.

[36] VALLES, B.; CASTILLO, E.; BERNAL, H. Rendimiento y degradabilidad ruminal de materia seca y energía de diez pastos tropicales cosechados a cuatro edades. Rev. Mex. Cien. Pec. 7 (2): 141-158. 2016.

[37] VILLANUEVA, P. C.; DÍAZ, E. V.; CHAY, C. A.; RAMÍREZ, A. L.; CASANOVA, L. F.; OROS, O. I. Comportamiento productivo e ingestivo de ovinos en crecimiento en sistemas silvopastoriles y de engorda en confinamiento. Rev. Mex. Cien. Pec. 10 (4): 870-884. 2019

[38] WENTZEL, H.; ALONSO, M. Factores que influyen en la utilización de especies leñosas por bovinos y ovinos en sistemas pastoriles. Praderas y forrajes. Facultad de Ciencias Agrarias, Universidad Austral de Chile. Agro. Sur. 48 (1): 1-9. 2020.

[39] ZAMBRANO, C.; ALTUVE, E.; ZAMBRANO, L.; PARRAGA, C. Conducta de ovinos a pastoreo en sistema silvopastoril tradicional con predominio de Saman (Phitecellobium saman) y Guácimo (Guazuma ulmifolia). Rev. Unell. Cien. Tec. (Volumen especial): 29-34. 2010.

[40] ZAMPALIGRÉ, N.; DOSSA, L.H.; SCHLECHT, E. Contribution of browse to ruminant nutrition across three agroecological zones of Burkina Faso. J. Arid Environm. 95: 55-64. 2013.

[41] ZUCCARI, A.E.; FERNÁNDEZ, G.D.; SOLLAZZO, L.A. Evaluación del consumo y comportamiento ingestivo de ovejas bajo dos regimenes de pastoreo durante el mes de enero. Rev. Argen. Prod. Anim. 27 (Supl. 1.): 152-154. 2007. 